\title{
„Die Riester-Rente alleine kann keine Altersarmut verhindern"
}

\begin{abstract}
Der ehemalige Bundesminister für Arbeit und Sozialordnung, Walter Riester, im Gespräch mit WiSt-Redakteur
\end{abstract} Jörg Rieger

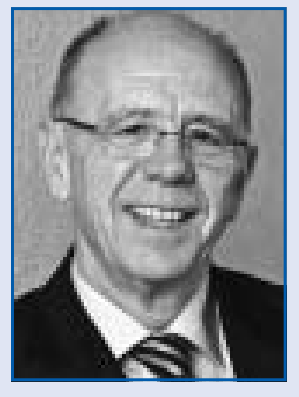

Walter Riester war von 1998 bis 2002 Bundesminister für Arbeit und Sozialordnung im Kabinett von Bundeskanzler Gerhard Schröder. Der gelernte Fliesenleger trat 1977 der IG Metall bei und stieg dort bis zum Zweiten Vorsitzenden auf. In dieser Zeit war Riester zudem Aufsichtsratmitglied in Wirtschaftsunternehmen wie Audi, Bosch und Heidelberg Druckmaschinen. Von 2002 bis 2009 war er für die SPD Mitglied des deutschen Bundestages.

Die Gesetzliche Rentenversicherung hat 2014 ihr 125jähriges Jubiläum gefeiert. Der erste Reichskanzler Otto von Bismarck hat sie im Jahr 1889 eingeführt. Die deutsche Rentenversicherung galt mit ihrem Umlageverfahren bis weit ins 20. Jahrhundert hinein als Erfolgsmodell. Durch den demographischen Wandel begann sie allerdings zu bröckeln. In Zukunft droht die gesetzliche Rente zu einer Art Grundsicherung zu verkommen, weshalb dringender Handlungsbedarf besteht. Doch nicht immer dreht die Politik an den richtigen Stellschrauben.

Im WiSt-Interview spricht Walter Riester über die falsche Finanzierung des Rentenpakets, die Vorzüge der nach ihm benannten Riester-Rente und das künftige Problem der Altersarmut in Deutschland.

Stichwörter: Altersarmut, Gesetzliche Rentenversicherung, Rentenpaket, Riesterrente, Umlageverfahren

Zum 1. Januar 2015 sind die Rentenbeiträge gesunken, während die Renten trotz einer stark alternden Bevölkerung zum 1. Juli dieses Jahres um 1 bis 2 Prozent steigen sollen. Leben wir in einem Wunderland?

Walter Riester: In einem Schlaraffenland leben wir definitiv nicht. Dennoch ist die Situation nur auf den ersten Blick absurd. Denn das Prinzip des Systems der Gesetzlichen Rentenversicherung ist so angelegt, dass es zu Leistungssteigerungen bzw. Beitragskürzungen kommt, wenn sich die Einnahmeseite verbessert darstellt. Genau dies ist in den letzten Jahren aufgrund von Lohnerhöhungen und zusätzlichen sozialversicherungspflichtigen Arbeitsplätzen geschehen. Eigentlich hätten die Beiträge bei Rentenreserven von mehr als $30 \mathrm{Mrd}$. Euro schon früher sinken müssen.

\section{Warum ist dies nicht passiert?}

Riester: Weil die Große Koalition zum 1. Juli 2014 das Rentenpaket in Kraft gesetzt hat. Sie ist drauf und dran, die Rentenreserven an einige wenige Jahrgänge zu verfrühstücken.

Die Befürworter des Pakets argumentieren, dass es eine Gerechtigkeitslücke schließt. Der Slogan heißt: Nicht geschenkt. Sondern verdient.

Riester: Dieser Slogan ist Humbug. Denn es werden letztlich einige wenige Jahrgänge, denen es auch im Rentenalter vergleichsweise gut geht, zu Lasten jüngerer Generationen begünstigt. Bei der sogenannten Mütterrente als ein Bestandteil des Rentenpakets versucht man, eine Gerechtigkeitslücke zu schließen, die sich Ende der 1990er Jahre aufgetan hat. Seinerzeit haben wir beschlossen, dass diejenigen, die nach 1992 oder später geborene Kinder erziehen, drei Jahre lang jeweils den vollen Rentenentgeltpunkt erhalten. Mehr war seinerzeit leider nicht zu machen; denn wir haben diese Leistung völlig zu Recht über Steuern finanziert.

Was also spricht dagegen, dass dies jetzt nachgeholt wird?

Riester: Sehr viel. Unsere Staatsverschuldung ist heute noch höher als um die Jahrtausendwende. Also ist man dazu übergegangen, das Ganze innerhalb des Systems zu finanzieren und die Rentenreserven anzutasten. Das ist Unsinn. Noch schlimmer stellt sich die Situation bei der Rente mit 63 dar.

Warum?

Riester: Ein Freund von mir, der in den Genuss dieser vorgezogenen, abschlagsfreien Rente gekommen ist, hat mir kürzlich erzählt, es fühle sich an, als hätte man einen Sechser im Lotto. Ich kann seine Sichtweise zwar gut verstehen. Dies ändert aber nichts daran, dass es sich um einen gravierenden Verstoß gegen die Teilhabeäquivalenz handelt. Man muss mit dem Gedanken aufräumen, dass die Rente eine Entschädigung für lebenslanges, schweres Arbeiten ist.

\section{Was sonst ist die gesetzliche Rente?}

Riester: Sie ist der Ausgleich für die Beitragszahlungen, die man Zeit seines Erwerbslebens in den Rententopf eingezahlt hat. Je höher diese Einzahlungen sind, desto mehr Rente gibt es. Fast jeder findet dieses Prinzip gut. Doch durch die Rente mit 63 wird es komplett ausgehebelt. Denn von heute auf morgen können ganz bestimmte Gruppen vorzeitig abschlagsfrei in Rente gehen. Mal angenom- 
men, ein Arbeitnehmer ist aus welchen Gründen auch immer - Arbeitslosigkeit, Krankheit, Studium - erst mit Anfang 20 ins Berufsleben eingestiegen. Er kann selbst bei einem anschließend lückenlosen Erwerbsleben nicht in den Genuss der abschlagsfreien Rente mit 63 kommen, obgleich er vielleicht sogar mehr Beiträge einzahlt. Das ist in meinen Augen nicht gerecht und erst recht nicht ökonomisch sinnvoll.

Prof. Raffelhüschen hat vor einiger Zeit gegenüber der WiSt gesagt, dass sogar die Rente mit 67 ein Geschenk ist, da wir in Wahrheit noch länger arbeiten müssten. Stimmen Sie dem zu?

Riester: Ja. Das Umlageverfahren ist in dieser Form 1957 in Deutschland eingeführt worden. Damals war die durchschnittliche Rentenbezugszeit knapp 10 Jahre - und auf dieser Grundlage wurde auch kalkuliert. Heute kann diese Generation damit rechnen, sogar 20 Jahre lang Rente zu beziehen. Für die derzeitigen Rentner ist das Umlageverfahren also ein sehr gutes Geschäft, für die heute 30- bis 50-Jährigen dagegen definitiv nicht mehr. Sie müssen länger arbeiten, mehr einbezahlen und werden am Ende aller Tage aufgrund des demographischen Wandels weniger Leistungen erhalten.

Ohne das Rentenpaket hätte man der Generationengerechtigkeit also besser Rechnung getragen...

Riester: Ja - und nicht nur das. Die Gesamtkosten des Rentenpakets belaufen sich nach Schätzungen der Bundesregierung auf $160 \mathrm{Mrd}$. Euro. Wissenschaftler vermuten, dass es am Ende $240 \mathrm{Mrd}$. Euro sein werden. Gehen wir mal von der goldenen Mitte aus: Mit 200 Mrd. Euro kann man sehr viel anstellen, um unser Land zu modernisieren und gerechter zu machen.

Immerhin galt die Rentenversicherung - anders als etwa die Kranken-oder Pflegeversicherung - als weitgehend saniert, auch wenn dies mit tiefen Einschnitten in der $\mathrm{Zu}$ kunft einhergeht...

Riester: Ich würde es eher mit „reformiert“ umschreiben. Da es sich bei einem Umlageverfahren um ein sehr dynamisches System handelt, kann man es nicht auf ewig sanieren. Man muss es immer wieder anpassen, wenn sich wichtige Stellschrauben ändern. Genau das haben wir in der rot-grünen Koalition unter Kanzler Gerhard Schröder getan.

Ihr Meisterstück war 2002 die Einführung der RiesterRente. Wie funktioniert sie?

Riester: Es handelt sich um eine ergänzende Altersvorsorge, die auf privaten Ersparnissen beruht und staatlich gefördert wird; zum einen durch eine nachgelagerte Besteuerung, zum anderen durch Zulagen. Die nachgelagerte Besteuerung ist nicht neu und kommt auch in vielen anderen Ländern zum Einsatz. Das Besondere an der Riester-Rente ist, dass sie sich nicht nur für Menschen mit vergleichsweise hohen Einkommen, sondern wegen der Zulagen eben auch für Geringverdiener lohnt. Alle eingezahlten Beiträge und die Zulagen sind garantiert. Ein solches System sucht weltweit seinesgleichen.
Können Sie uns das bitte an einigen Beispielen erläutern...

Riester: Man nehme einen alleinverdienenden Gutverdiener mit einem Jahreseinkommen von 55.000 Euro. Er kann bis zu 2.100 Euro, die er in einen Riester-Vertrag einzahlt, von der Steuer absetzen. Bei einem Spitzensteuersatz inklusive Soli-Zuschlag von derzeit 44,31 Prozent, beträgt die Steuerersparnis 930,51 Euro jährlich. Eine durchschnittlich verdienende Familie mit zwei kleinen Kindern profitiert mit einem noch viel größeren Wirkungsgrad. Denn sie erhält die Zulagen, da diese höher sind als die Steuerersparnis. Die Zulagen betragen zusammen 908 Euro. Bei einem angenommenen Haushaltseinkommen von 30.000 Euro muss die Familie 1.200 Euro jährlich in den Riester-Vertrag einzahlen. Durch die Zulagen beträgt der Eigenanteil nur noch 292 Euro. Eine geringverdienende Alleinerziehende mit zwei Kindern muss sogar nur den Mindestbeitrag von 60 Euro einzahlen und erhält jährlich einen staatlichen Zuschuss von 754 Euro. Das ist doch ein toller Hebeleffekt.

Dennoch stagniert die Zahl der abgeschlossenen Verträge seit einigen Jahren bei 16 Millionen, während das Potenzial mittlerweile bei über 40 Millionen liegen dürfte...

Riester: Das liegt meines Erachtens an der anhaltenden Kritik der Medien an der Riester-Rente. Das DIW (Anm. der Red.: Deutsches Institut für Wirtschaftsforschung) hat dieser Form der Altersvorsorge einen Bärendienst erwiesen, als es den Sparern empfohlen hat, das Geld lieber in einen Sparstrumpf als in solch ein Produkt zu stecken. Viele Medien haben das aufgegriffen - mit fatalen Folgen...

\section{...oftmals werden die hohen Gebühren kritisiert...}

Riester: Dem kann ich im Großen und Ganzen widersprechen. Natürlich gibt es wie überall auch hier unter den Anbietern schwarze Schafe. Meist unterscheiden sich die Kosten von Riester-Produkten und normalen Verträgen jedoch nicht - und wenn, dann ist die Differenz nur ganz gering. Dabei ist der Verwaltungsaufwand bei den Riester-Verträgen wegen der Beantragung der Zulagen deutlich höher.

Die Versicherungsverträge machen den Großteil der Riester-Verträge aus. Gerade diese Produkte verschlingen in den ersten Jahren hohe Gebühren...

Riester: Ja, aber das ist bei normalen Lebensversicherungen doch ganz genauso. Deshalb ist es ratsam, die Verträge so früh wie möglich abzuschließen und nicht vorzeitig zu kündigen. Dann verteilen sich die Kosten über die Zeit und fallen nicht so stark ins Gewicht.

Auch das anhaltend geringe Zinsniveau macht den Versicherungen und damit auch deren Kunden zu schaffen. Der Garantiezins ist zum 1. Januar auf nur noch 1,25 Prozentpunkte gefallen.

Riester: Über längere Zeiträume wechseln sich Phasen hoher und niedriger Zinsen ab. Insofern gilt das Argument der langfristigen Anlagehorizonte auch hier, wenngleich ich davon ausgehe, dass das niedrige Zinsumfeld noch länger anhalten dürfte. Doch man hat ja auch die Möglichkeit 
auf den Wohn-Riester oder riestergeförderte Fondssparpläne zu setzen. Vor allem die Abschlüsse von geförderten Bausparverträgen haben zuletzt sprunghaft zugenommen.

Die Tatsache, dass die Versicherungen und Fondsgesellschaften die eingezahlten Beiträge nebst Zulagen am Laufzeitende garantieren müssen, muss doch zu Lasten der Rendite gehen.

Riester: Nein, nicht zwangsläufig. Wie schon geschildert, sind die Kosten zwischen riester-geförderten und normalen Verträgen oftmals identisch, auch bei einem und demselben Fonds. Folglich hat die Garantie keinen Einfluss auf die Rendite.

Man muss jedoch konstatieren, dass die Riester-Renten in vielen Fällen sehr klein sein werden, häufig nicht viel mehr als 100 Euro und da ist die Inflation noch gar nicht berücksichtigt.

Riester: Das hängt davon ab, wie viel man Zeit seines Lebens in die Verträge einzahlt. Aber natürlich darf man von der Riester-Rente auch keine Wunderdinge erwarten. Sie ist schließlich als ergänzende Altersvorsorge konzipiert worden und kann alleine natürlich auch keine Altersarmut verhindern.

Damit werden wir allerdings in Deutschland zunehmend fertig werden müssen.

Riester: Da stimme ich Ihnen zu. Der im letzten Jahrzehnt entstandene Niedriglohnsektor und die starke Zunahme von Teilzeit-Beschäftigten werden früher oder später dazu führen, dass die Rente trotz Riestern für einen immer gröBeren Teil der Bevölkerung nicht mehr zum Leben reicht.

CDU/CSU und SPD haben die sogenannte Lebensleistungsrente im Koalitionsvertrag festschreiben lassen, um dieser Entwicklung vorzubeugen. Doch um sie ist es relativ still geworden.

Riester: Ich finde die Idee grundsätzlich gut, auch wenn mir den Begriff überhaupt nicht gefällt. Denn Lebensleistungsrente suggeriert wieder, dass man die gesetzliche Rente als Entschädigung für die Anstrengungen während des Erwerbslebens erhält. Wir bekommen sie aber aufgrund unserer eingezahlten Beträge. Wenn man von diesem Äquivalenzprinzip abweichen möchte, bedarf es einer guten Begründung. Sehr niedrige Renten aufzustocken ist sicherlich ein Argument, auch wenn es steuerfinanziert sein muss. Gleichzeitig gebe ich jedoch zu bedenken, dass die wachsende Gruppe der Selbstständigen zu einem groBen Teil nicht von einer wie auch immer ausgestaltenden Lebensleistungsrente profitieren würde.

Genauso wenig wie von der Riester-Rente. Warum hat man diese Gruppe links liegengelassen?

Riester: Das hat man nicht. Doch unser System ist historisch so gewachsen. Darin unterscheiden wir uns von anderen Ländern. Letztlich geht es auf Bismarck zurück, dass in der Gesetzlichen Rentenversicherung nur die abhängig Beschäftigten pflichtversichert sind. Den Unternehmern hat man zugetraut, dass sie eigenständig fürs Alter vorsorgen. Diese Zweiteilung hat sich auch über Jahrzehnte bewährt. Doch heute müssen wir feststellen, dass sich die Situation geändert hat. Die Zahl der (Schein-)Selbständigen, die zu wenig Altersvorsorge betreiben, wächst immer weiter an. Dem muss man entgegenwirken. Ansonsten haben wir in ein paar Jahren ein massives Problem. Mir ist aber schon bewusst, dass man an dieser Stelle dicke Bretter bohren muss. Schließlich will jede Selbstständigen-Gruppe ihre Pfründe verteidigen.

Herr Riester, vielen Dank für das Gespräch! 